\title{
PROBABILITY STRUCTURE PRESERVING AND ABSOLUTE CONTINUITY
}

\author{
Yaozhong HU \\ Department of Mathematics, University of Kansas, 405 Snow Hall, Lawrence, KS 66045-2142, USA \\ Received 13 December 2000, revised 9 May 2001
}

ABSTRACT. - The concept of probability structure preserving mapping is introduced. The idea is applied to define stochastic integral for fractional Brownian motion (fBm) and to obtain an anticipative Girsanov theorem for fBm. @ 2002 Éditions scientifiques et médicales Elsevier SAS

AMS classification: $60 \mathrm{H} 05 ; 60 \mathrm{H} 07 ; 60 \mathrm{H} 15 ; 60 \mathrm{~B} 11 ; 28 \mathrm{~B} 20$

Keywords: Fractional Brownian motions; Probability structure preserving mapping; Fractional calculus; Stochastic calculus; Translation; Radon-Nikodym derivative

RÉSUMÉ. - Un concept d'application préservant la probabilité est introduit. L'idée est appliquée à la définition de l'intégrale stochastique pour le mouvement brownien fractionnaire et à la définition d'une formule de Girsanov anticipante. ( 2002 Éditions scientifiques et médicales Elsevier SAS

\section{Introduction}

Fractional Brownian motion ( $\mathrm{fBm}$ ) has received a great deal of attention in recent years. Various authors have developed stochastic calculus applicable to fBm (see for example, $[1,3-5,10]$ for recent development).

This paper is motivated by the problem of absolute continuity for $\mathrm{fBm}$ with respect to its translation. Let $\Omega$ be the space of real valued continuous functions $\omega(t), t \in \mathbb{R}$, with $\omega(0)=0$. Define

$$
D\left(\omega_{1}, \omega_{2}\right)=\sum_{n=1}^{\infty} \frac{1}{2^{n}} \frac{\sup _{-n \leqslant t \leqslant n}\left|\omega_{1}(t)-\omega_{2}(t)\right|}{1+\sup _{-n \leqslant t \leqslant n}\left|\omega_{1}(t)-\omega_{2}(t)\right|} .
$$

Then it is easy to see that $D$ is a metric on $\Omega$ and $(\Omega, D)$ is a polish space (see [20]). Let $\mathcal{F}$ be the Borel $\sigma$-algebra of $\Omega$. For any given number $H \in(0,1)$, there is a probability

\footnotetext{
This work is supported in part by the National Science Foundation under Grant No. EPS-9874732, matching support from the State of Kansas and General Research Fund of the University of Kansas.

E-mail address: hu@math.ukans.edu (Y. Hu).
} 
measure $P^{H}$ on $(\Omega, \mathcal{F})$ such that $B_{t}^{H}: \Omega \rightarrow \mathbb{R}, t \in \mathbb{R}$, defined by $B_{t}^{H}(\omega)=\omega(t), t \in \mathbb{R}$, is a fractional Brownian motion with Hurst parameter $H$. Namely, $\left(B_{t}^{H}, t \in \mathbb{R}\right)$ is a Gaussian process with mean 0, and covariance

$$
\mathbb{E}\left(B_{t}^{H} B_{s}^{H}\right)=c_{H}\left(|t|^{2 H}+|s|^{2 H}-|t-s|^{2 H}\right),
$$

where $c_{H}=\frac{\Gamma(2-2 H) \cos (\pi H)}{\pi H(1-H)}$. We shall call $\left(\Omega, \mathcal{F}, P^{H}\right)$ the canonical fractional Wiener space with Hurst parameter $H$. The expectation on this probability space is denoted by $\mathbb{E}^{H}$. It is customary to denote the element of $\Omega$ by $B^{H}$. When $H=1 / 2$, we obtain the (usual) canonical Wiener space and we will omit the dependence on $H$ when $H=1 / 2$.

Now let $\Lambda$ be a transform from $\left(\Omega, \mathcal{F}, P^{H}\right)$ to $\left(\Omega, \mathcal{F}, P^{H}\right)$ defined by

$$
B^{H} \rightarrow B^{H}+\int_{0} g(s) \mathrm{d} s,
$$

where $g$ is an anticipative stochastic process. Under suitable conditions, $\Lambda$ induces another probability measure $P^{H} \circ \Lambda^{-1}$ on $(\Omega, \mathcal{F})$ given by

$$
P^{H} \circ \Lambda^{-1}(A)=P^{H}\left(\Lambda^{-1}(A)\right)=P^{H}(\{\omega, \Lambda(\omega) \in A\}), \quad \forall A \in \mathcal{F} .
$$

We are interested in the problem of absolute continuity of $P^{H} \circ \Lambda^{-1}$ with respect to $P^{H}$.

In the classical Brownian motion case (i.e., when $H=1 / 2$ ) this problem has been studied by many authors. We refer to $[2,21]$ and in particular the references therein.

In the general fractional Brownian motion case $(H \neq 1 / 2)$, this problem has been studied extensively when $g$ is a deterministic function (see [11] and the references therein).

This paper studies the general (anticipative) case. We obtain a general theorem about the absolute continuity and a general formula for the Radon-Nikodym density.

Presumably, we may utilize the general formula of Ramer-Kusuoka and compute the Carleman-Fredholm determinant appeared in the Ramer-Kusuoka formula. This has been the idea for example in [2,21] in the classical Brownian motion case. However, this paper develops another method which makes use of the known results in the classical Brownian motion case.

By a theorem (see Theorem 2.1 below) in the framework of measure theory, it is known that if we can find a measure-preserving one-to-one mapping $T$ from $(\Omega, \mathcal{F}, P)$ to $\left(\Omega, \mathcal{F}, P^{H}\right)$ and a measurable mapping $\Gamma$ from $\Omega$ to $\Omega$ such that $\Lambda \circ T=T \circ \Gamma$. Then

$$
\frac{\mathrm{d} P^{H} \circ \Lambda^{-1}}{\mathrm{~d} P^{H}}=\frac{\mathrm{d} P \circ \Gamma^{-1}}{\mathrm{~d} P} \circ T^{-1}
$$

as long as we know that $\frac{\mathrm{d} P \circ \Gamma^{-1}}{\mathrm{~d} P}$ exists.

It is interesting to directly construct a mapping $T$ from $(\Omega, \mathcal{F}, P)$ to $\left(\Omega, \mathcal{F}, P^{H}\right)$ and a mapping $\Gamma$ from $(\Omega, \mathcal{F}, P)$ to itself such that $\Lambda \circ T=T \circ \Gamma$. We may then obtain the Radon-Nikodym derivative by using (1.3) since there is an extensive study on the computation of $\frac{\mathrm{d} P \circ \Gamma^{-1}}{\mathrm{~d} P}$ in the classical Brownian motion case. 
However, we will extend (1.3) to a more general case. We introduce the concept of probability structure preserving mapping $V$ from the set of measurable functions on $(\Omega, \mathcal{F}, P)$ to the set of measurable functions on $\left(\Omega, \mathcal{F}, P^{H}\right)$. $\Gamma$ (and $\Lambda$ ) can be considered as transform which maps a function on $(\Omega, \mathcal{F}, P)$ (and on $\left(\Omega, \mathcal{F}, P^{H}\right)$ ) to another function on $(\Omega, \mathcal{F}, P)$ (and on $\left(\Omega, \mathcal{F}, P^{H}\right)$ ). We shall prove that if $\Lambda \circ V=$ $V \circ \Gamma$, then

$$
\frac{\mathrm{d} P^{H} \circ \Lambda^{-1}}{\mathrm{~d} P^{H}}=V \circ \frac{\mathrm{d} P \circ \Gamma^{-1}}{\mathrm{~d} P} .
$$

This is an extension of (1.3). From this result and a result on the Radon-Nikodym derivative for the classical Brownian motion, we obtain a formula for the RadonNikodym derivative for the fractional Brownian motion.

The idea of the probability structure preserving mapping may carry many other established results on one probability space to another probability space. We do not claim that all results on classical Brownian motion may be extended to fractional Brownian motion by using this idea. However, many other results on the classical Brownian motion may also be extended to the fractional Brownian motion case through this probability structure preserving mapping.

The probability structure preserving mapping $V$ introduced in this paper is similar to a correspondence introduced by the author and his adviser, Prof. P.A. Meyer in [7-9]. However, in that correspondence the multiple Stratonovich integrals play an important role. In the mapping considered in this paper, the similar role is played by multiple Itô type integrals.

In Section 2, we give the definition of the probability structure preserving mapping $V$ and prove that this mapping may be defined for any measurable function. We also present a general way to construct this mapping for abstract Wiener space case.

In Section 3, we introduce a particular probability structure preserving mapping $V$ between Brownian motion and fractional Brownian motion with Hurst parameter $H$ and establish some useful properties of $V$. We use this mapping and the definition of stochastic integral for Brownian motion to define stochastic integral for fractional Brownian motion.

In Section 4, we apply the probability structure preserving mapping $V$ introduced in Section 3 to obtain a Girsanov type theorem for fractional Brownian motion.

\section{Probability structure preserving}

Let $\left(\Omega_{1}, \mathcal{F}_{1}, P_{1}\right)$ and $\left(\Omega_{2}, \mathcal{F}_{2}, P_{2}\right)$ be two measurable spaces. Let $T$ be an invertible measurable mapping from $\Omega_{1}$ to $\Omega_{2}$ such that

$$
\int_{\Omega_{1}} F \circ T\left(\omega_{1}\right) P_{1}\left(\mathrm{~d} \omega_{1}\right)=\int_{\Omega_{2}} F\left(\omega_{2}\right) P_{2}\left(\mathrm{~d} \omega_{2}\right)
$$

for all bounded measurable function $F: \Omega \rightarrow \Omega$. Namely, $T$ is a measure-preserving one to one transform. 
THEOREM 2.1. - Let $\Gamma$ be a measurable transformation from $\Omega_{1}$ to itself and let $\Lambda$ be a measurable transformation from $\Omega_{2}$ to itself. Assume that the following diagram

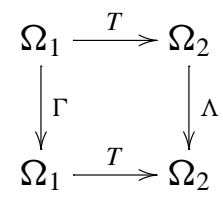

commutes. Then

$$
\frac{\mathrm{d} P_{2} \circ \Lambda^{-1}}{\mathrm{~d} P_{2}}=\frac{\mathrm{d} P_{1} \circ \Gamma^{-1}}{\mathrm{~d} P_{1}} \circ T^{-1}
$$

Proof. - This result may be known. However, I could not find it in a standard reference. I will sketch a simple proof.

Let $A \in \mathcal{F}_{2}$ and $B=T^{-1}(A) \in \mathcal{F}_{1}$. Then

$$
\begin{aligned}
P_{2} \Lambda^{-1}(A) & =P_{2}\left(\Lambda^{-1} T(B)\right)=P_{2}\left(T \Gamma^{-1}(B)\right)=P_{1}\left(\Gamma^{-1}(B)\right) \\
& =\int_{B} \frac{\mathrm{d} P_{1} \circ \Gamma^{-1}}{\mathrm{~d} P_{1}}(x) P_{1}(\mathrm{~d} x)=\int_{A} \frac{\mathrm{d} P_{1} \circ \Gamma^{-1}}{\mathrm{~d} P_{1}}\left(T^{-1} y\right) P_{2}(\mathrm{~d} y) .
\end{aligned}
$$

This proves (2.2).

Let $(\Omega, H, P)$ be an abstract Wiener space, where $H$ is a Hilbert space with scalar product $\langle\cdot, \cdot\rangle_{H}$. There is no ambiguity to use $H$ both for the Hurst parameter and for a Hilbert space. For any $h \in H$, there is a Gaussian random variable, denoted by $\langle\omega, h\rangle$, such that it has mean 0 and covariance

$$
\mathbb{E}\left(\left\langle\cdot, h_{1}\right\rangle\left\langle\cdot, h_{2}\right\rangle\right)=\left\langle h_{1}, h_{2}\right\rangle_{H} .
$$

Define $\mathcal{F}$ the smallest $\sigma$-algebra such that for all $h \in H,\langle\omega, h\rangle$ is $\mathcal{F}$-measurable. Let $h \in H$. Then $F(\omega)=\exp (\langle\omega, h\rangle)$ is called an exponential functional or an exponential vector. Let $\mathcal{E}=\mathcal{E}(\Omega, \mathcal{F}, P)$ be the space of finite linear combination of the exponential functionals (abbreviated as FLICEF). Then $\mathcal{E}$ is a linear space which is closed with respect to multiplication, i.e., if $F, G \in \mathcal{E}$, then $F G \in \mathcal{E}$. $\mathcal{E}$ is also dense in $L^{p}(\Omega, \mathcal{F}, P)$ for any $p \in[1, \infty)$.

Let $(\widetilde{\Omega}, \widetilde{H}, \widetilde{P})$ be another abstract Wiener space. Let $\widetilde{\mathcal{E}}$ be the space of FLICEF on $(\widetilde{\Omega}, \widetilde{\mathcal{F}}, \widetilde{P})$.

Definition 2.2.- A mapping $V$ from $\mathcal{E}$ to $\widetilde{\mathcal{E}}$ is called probability structure preserving mapping if

$$
\begin{aligned}
V(F+G) & =V(F)+V(G), \quad \forall F, G \in \mathcal{E}, \\
V(F G) & =V(F) V(G), \quad \forall F, G \in \mathcal{E}, \\
\mathbb{E}[F] & =\widetilde{\mathbb{E}}[V F], \quad \forall F \in \mathcal{E},
\end{aligned}
$$

where $\mathbb{E}$ and $\widetilde{\mathbb{E}}$ denote the expectations on the probability spaces $(\Omega, \mathcal{F}, P)$ and $(\widetilde{\Omega}, \widetilde{H}, \widetilde{P})$, respectively. 
The correspondence introduced in [7-9] satisfies the properties (2.3)-(2.4). However, it does not satisfy (2.5).

Example 2.3. - If $T$ is an invertible measurable mapping from $\Omega$ to $\widetilde{\Omega}$ and if $\widetilde{P}=$ $P \circ T^{-1}$, then $V F:=F \circ T^{-1}$ (where $F \in \mathcal{E}$ ) is a the probability structure preserving mapping.

It is clear that when $V$ is given by an invertible measurable mapping then $V$ is defined for all measurable function $F$ and $V(F G)=(V F)(V G)$.

Now we will show that in general case it is also true, i.e., $V$ can be defined for all measurable function $F$ and $V(F G)=(V F)(V G)$.

LEMMA 2.4. - If $F \in L^{2}(\Omega, \mathcal{F}, P)$, then $V F$ is well-defined.

Proof. - Let $\left\{F_{n}, n \geqslant 1\right\}$ be a sequence in $\mathcal{E}$ with the property that $F_{n} \rightarrow F$ in $L^{2}(\Omega, \mathcal{F}, P)$. Then for any $n, m \geqslant 1$, we have

$$
\begin{aligned}
\widetilde{\mathbb{E}}\left|V F_{n}-V F_{m}\right|^{2} & =\widetilde{\mathbb{E}}\left[\left(V F_{n}\right)^{2}+\left(V F_{m}\right)^{2}-2 V F_{n} V F_{m}\right] \\
& =\widetilde{\mathbb{E}}\left[V\left(F_{n}\right)^{2}+V\left(F_{m}\right)^{2}-2 V\left(F_{n} F_{m}\right)\right] \\
& =\widetilde{\mathbb{E}}\left[V\left(F_{n}^{2}+F_{m}^{2}-2 F_{n} F_{m}\right)\right] \\
& =\widetilde{\mathbb{E}}\left[\left(F_{n}^{2}+F_{m}^{2}-2 F_{n} F_{m}\right)\right] \\
& =\mathbb{E}\left(F_{n}-F_{m}\right)^{2} .
\end{aligned}
$$

Thus $\left\{V F_{n}, n \geqslant 1\right\}$ is a Cauchy sequence in $L^{2}(\widetilde{\Omega}, \widetilde{\mathcal{F}}, \widetilde{P})$. It is also easy to see that the limit is independent of the choice of $\left\{V F_{n}, n \geqslant 1\right\}$. Therefore the limit of the sequence $\left\{V F_{n}, n \geqslant 1\right\}$ can be defined as $V F$.

Lemma 2.5. - If $F, G \in L^{\infty}(\Omega, \mathcal{F}, P)$, then

$$
V(F G)=(V F)(V G) .
$$

Proof. - The assumption of the lemma implies that $F$ and $G$ are in $L^{2}(\Omega, \mathcal{F}, P)$. Thus $V(F)$ and $V(G)$ are well-defined by Lemma 2.4. Since $F$ and $G$ are in $L^{4}(\Omega, \mathcal{F}, P)$, there are sequences $\left\{F_{n}, n \geqslant 1\right\} \subset \mathcal{E}$ and $\left\{G_{n}, n \geqslant 1\right\} \subset \mathcal{E}$ such that

$$
\lim _{n \rightarrow \infty} \mathbb{E}\left|F_{n}-F\right|^{4}=\lim _{n \rightarrow \infty} \mathbb{E}\left|G_{n}-G\right|^{4}=0 .
$$

Similar to the proof of Lemma 2.4, we obtain

$$
\begin{aligned}
\widetilde{\mathbb{E}}\left|V\left(F_{n} G_{n}\right)-V(F G)\right|^{2} & =\mathbb{E}\left|F_{n} G_{n}-F G\right|^{2} \\
& \leqslant\left(\mathbb{E}\left|F_{n}\right|^{4}\right)^{1 / 2}\left(\mathbb{E}\left|G_{n}-G\right|^{4}\right)^{1 / 2}+\left(\mathbb{E}|G|^{4}\right)^{1 / 2}\left(\mathbb{E}\left|F_{n}-F\right|^{4}\right)^{1 / 2} \\
& \rightarrow 0 \quad(n \rightarrow \infty) .
\end{aligned}
$$

Thus there is a subsequence (without loss of generality we may choose the sequence itself) such that $V\left(F_{n} G_{n}\right)$ converges to $V(F G)$ almost surely as $n \rightarrow \infty$. On the other hand, since $V\left(F_{n}\right) \rightarrow V(F)$ and $V\left(G_{n}\right) \rightarrow V(G)$ in $L^{2}(\Omega, \mathcal{F}, \widetilde{P})$ we can find a subsequence such that $V\left(F_{n_{k}}\right) \rightarrow V(F)$ and $V\left(G_{n_{k}}\right) \rightarrow V(G)$ almost surely. 
Then $V\left(F_{n} G_{n}\right)=V\left(F_{n_{k}}\right) V\left(G_{n_{k}}\right) \rightarrow V(F) V(G)$ almost surely. Consequently, we have $V(F G)=V(F) V(G)$.

LEMMA 2.6. - If $F \in L^{\infty}(\Omega, \mathcal{F}, P)$ and if $f \rightarrow \mathbb{R}$ is a continuous function, then

$$
V f(F)=f(V F) .
$$

Proof. - Let $M>0$ be such that $|F| \leqslant M$ a.s. By the Weierstrass approximation theorem, there is a sequence of polynomials $\left\{P_{n}(x), n \geqslant 1\right\}$ such that $P_{n}$ converges uniformly to $f(x)$ on $[-M, M]$. By Lemma 2.5 it is easy to see that $V P_{n}(F)=P_{n}(V F)$. Since $P_{n}$ converges to $f, V P_{n}(F)=P_{n}(V F)$ converges to $f(V F)$ a.s. on the event $\{|V F| \leqslant K\}$ for any $K>0$. Letting $K \rightarrow \infty$, we see that $V P_{n}(F)$ converges to $f(V F)$ a.s. On the other hand,

$$
\mathbb{E}\left|V\left(P_{n}(F)\right)-V(f(F))\right|^{2}=\mathbb{E}\left|P_{n}(F)-f(F)\right|^{2} \rightarrow 0 \quad(n \rightarrow \infty) .
$$

This proves the lemma easily.

Lemma 2.7. - Let $F \in L^{\infty}(\Omega, \mathcal{F}, P)$ and let $f$ be a continuous function. Then for any $\varepsilon \in \mathbb{R}$,

$$
\widetilde{P}(f(V F) \geqslant \varepsilon)=P(f(F) \geqslant \varepsilon) .
$$

Proof. - Notice that $g_{n, \varepsilon}:=\mathrm{e}^{-n(\varepsilon-f(x))^{+}}$is a continuous function of $x$, where $a^{+}$ denotes the positive part of $a$ and that

$$
\lim _{n \rightarrow \infty} g_{n, \varepsilon}(x)=I_{\{f(x) \geqslant \varepsilon\}} \quad \forall x \in \mathbb{R},
$$

where $I$ denotes the indicate function. Then

$$
\begin{aligned}
\widetilde{P}(f(V F) \geqslant \varepsilon) & =\widetilde{\mathbb{E}} I_{\{f(V F) \geqslant \varepsilon\}}=\widetilde{\mathbb{E}} \lim _{n \rightarrow \infty} \mathrm{e}^{-n(\varepsilon-f(V F))^{+}} \\
& =\lim _{n \rightarrow \infty} \widetilde{\mathbb{E}} \mathrm{e}^{-n(\varepsilon-f(V F))^{+}}=\lim _{n \rightarrow \infty} \widetilde{\mathbb{E}} V \mathrm{e}^{-n(\varepsilon-f(F))^{+}} \\
& =\lim _{n \rightarrow \infty} \mathbb{E} \mathrm{e}^{-n(\varepsilon-f(F))^{+}}=\mathbb{E} \lim _{n \rightarrow \infty} \mathrm{e}^{-n(\varepsilon-f(F))^{+}} \\
& =\mathbb{E} I_{\{f(F) \geqslant \varepsilon\}}=P(f(F) \geqslant \varepsilon) .
\end{aligned}
$$

This proves the lemma.

Now we are ready to state and prove the main theorem of this section.

THEOREM 2.8. - Let $F: \Omega \rightarrow \mathbb{R}$ be measurable and $F<\infty$ a.s.

(i) If $F_{n} \rightarrow F$ in probability, where $F_{n} \in L^{\infty}(\Omega, \mathcal{F}, P)$, then $V F_{n}$ converges in probability. The limit in probability of $V F_{n}$ is defined as $V F$.

(ii) If $f$ is continuous, then

$$
V f(F)=f(V F) .
$$

Proof. - (i) From Lemma 2.7, it follows that

$$
\widetilde{P}\left(\left|V F_{m}-V F_{m}\right| \geqslant \varepsilon\right)=\widetilde{P}\left(\left|V\left(F_{m}-F_{m}\right)\right| \geqslant \varepsilon\right)=P\left(\left|F_{m}-F_{m}\right| \geqslant \varepsilon\right) .
$$


This shows that $\left\{V F_{n}, n \geqslant 1\right\}$ is a Cauchy sequence with respect to the convergence in probability. The unique limit of this sequence is independent of the choice of $\left\{V F_{n}, n \geqslant 1\right\}$. This limit is defined as $V F$.

(ii) Now let $F_{n}=F I_{\{|F| \leqslant n\}}$. Since $V F_{n} \rightarrow V F$ in probability, then there is a subsequence $n_{k}$ such that $V F_{n_{k}} \rightarrow V F$ a.s. Thus

$$
V\left(f\left(F_{n_{k}}\right)\right)=f\left(V F_{n_{k}}\right) \rightarrow f(V F) \quad \text { a.s. }
$$

Let $K>0$ be a given arbitrary number. Since $f$ is uniformly continuous on $[-K, K]$, there is a $\delta>0$ such that $|f(x)-f(y)| \leqslant \varepsilon$ for all $x, y \in[-K, K]$ with $|x-y|<\delta$. By Lemma 2.7, it follows that

$$
\begin{aligned}
\widetilde{P}\left(\left|V\left(f\left(F_{n}\right)\right)-V(f(F))\right| \geqslant \varepsilon\right)= & P\left(\left|f\left(F_{n}\right)-f(F)\right| \geqslant \varepsilon\right) \\
= & P\left(\left\{\left|f\left(F_{n}\right)-f(F)\right| \geqslant \varepsilon\right\} \cap\{|F| \leqslant K\}\right) \\
& +P\left(\left\{\left|f\left(F_{n}\right)-f(F)\right| \geqslant \varepsilon\right\} \cap\{|F|>K\}\right) \\
\leqslant & \left.P\left(\left\{\mid F_{n}\right)-F \mid \geqslant \delta\right\}\right)+P(\{|F|>K\}) .
\end{aligned}
$$

From this inequality it follows that $V\left(f\left(F_{n}\right)\right) \rightarrow V(f(F))$ in probability. When combined with (2.8), this implies the second part of the theorem.

Remark 2.9. - By Theorem 2.8, we shall call $V$ a probability structure preserving mapping from $(\Omega, \mathcal{F}, P)$ to $(\widetilde{\Omega}, \widetilde{\mathcal{F}}, \widetilde{P})$.

COROLlary 2.10. $-V$ is injective.

Proof. - Let $F$ be measurable such that $V F=0$ a.s. Then

$$
P(|F|>0)=\widetilde{P}(|V F|>0)=0 .
$$

This implies that $F=0$ a.s.

It is interesting to know the general conditions under which a probability structure preserving mapping is given by a measurable transform $T$, i.e., $V \circ F=F \circ T$.

Now let $\Gamma$ be an invertible measurable mapping from $\Omega$ to itself and let $\Lambda$ be an invertible measurable mapping from $\widetilde{\Omega}$ to itself. $\Gamma$ induces a mapping from $\mathcal{E}$ to another functional space $\mathcal{B}$. This mapping is still denoted by $\Gamma$. Namely, $(\Gamma F)(\omega)=F \circ \Gamma(\omega)$, for all $F \in \mathcal{E}$ and $\omega \in \Omega$. Let $\Lambda$ be the corresponding induced mapping from $\widetilde{\mathcal{E}}$ to $\tilde{\mathcal{B}}$. We are going to establish

THEOREM 2.11. - Let $V$ be a probability structure preserving mapping from $(\Omega, \mathcal{F}, P)$ to $(\widetilde{\Omega}, \widetilde{\mathcal{F}}, \widetilde{P})$. Let $\Gamma$ (and $\Lambda$ ) be invertible measurable mapping from $\Omega$ (and $\widetilde{\Omega}$ ) to themselves. Assume that the following diagram

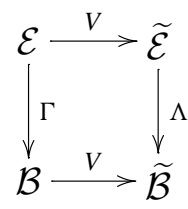


commutes. If $P \circ \Gamma^{-1}$ is absolutely continuous with respect to $P$, then $\widetilde{P} \circ \Lambda^{-1}$ is absolutely continuous with respect to $\widetilde{P}$. Moreover, the following identity holds:

$$
\frac{\mathrm{d} \widetilde{P} \circ \Lambda^{-1}}{\mathrm{~d} \widetilde{P}}=V \circ\left(\frac{\mathrm{d} P \circ \Gamma^{-1}}{\mathrm{~d} P}\right) .
$$

Proof. - Assume that $P \circ \Gamma^{-1}$ is absolutely continuous with respect to $P$. Let $G \in \widetilde{\mathcal{E}}$ and denote $F=V^{-1} G$. By the commutativity of the diagram (2.10) we obtain that

$$
G \circ \Lambda=V \circ(F \circ \Gamma) .
$$

Denote

$$
R_{1}(x)=\frac{\mathrm{d} \widetilde{P} \circ \Gamma^{-1}}{\mathrm{~d} \widetilde{P}}(x), \quad x \in \Omega .
$$

From the probability structure preserving property of $V$, it follows that

$$
\begin{aligned}
\int_{\widetilde{\Omega}} G \circ \Lambda(y) \widetilde{P}(\mathrm{~d} y) & =\int_{\Omega} F \circ \Gamma(x) P(\mathrm{~d} x)=\int_{\Omega} F(x) R_{1}(x) P(\mathrm{~d} x) \\
& =\int_{\widetilde{\Omega}} V\left(F R_{1}\right)(y) \widetilde{P}(\mathrm{~d} y)=\int_{\widetilde{\Omega}}(V F)(y)\left(V R_{1}\right)(y) \widetilde{P}(\mathrm{~d} y) \\
& =\int_{\widetilde{\Omega}} G(y)\left(V R_{1}\right)(y) \widetilde{P}(\mathrm{~d} y) .
\end{aligned}
$$

This yields that

$$
\frac{\mathrm{d} \widetilde{P} \circ \Lambda^{-1}}{\mathrm{~d} \widetilde{P}}(y)=\left(V R_{1}\right)(y), \quad \text { for a.s. } y \in \widetilde{\Omega},
$$

proving the theorem.

Multiple stochastic integral over a Wiener space is well-defined (see [6] and the references therein). Let us recall that any element of $L^{2}(\Omega, \mathcal{F}, P)$ can be represented by its chaos expansion:

$$
F=\sum_{n=0}^{\infty} \frac{1}{n !} I_{n}\left(f_{n}\right), \quad \text { with } \sum_{n=0}^{\infty} \frac{1}{n !}\left\|f_{n}\right\|_{H^{\otimes n}}^{2}<\infty
$$

where $f_{n} \in H^{\otimes n}$ (the symmetric tensor product Hilbert space over $H$ ) and $I_{n}$ is the multiple Itô type multiple stochastic integral. It is known that

$$
\mathbb{E}\left(F^{2}\right)=\sum_{n=0}^{\infty} \frac{1}{n !}\left\|f_{n}\right\|_{H^{\otimes n}}^{2}<\infty .
$$

The Fock space over $H$ is a Hilbert space defined by

$$
\Phi(H)=\left\{f=\left(f_{0}, f_{1}, \ldots, f_{n}, \ldots\right), f_{n} \in H^{\otimes n}\right\}
$$


with the Hilbert norm

$$
\|f\|_{\Phi(H)}^{2}=\sum_{n=0}^{\infty} \frac{1}{n !}\left\|f_{n}\right\|_{H^{\otimes n}}^{2} .
$$

Thus we have an isometry between $L^{2}(\Omega, \mathcal{F}, P)$ and the Fock space $\Phi(H)$ over the Hilbert space $H$ (see $[6,12,16]$, and the references therein for more detail).

Let $\rho$ be a one-to-one mapping from $H$ to $\widetilde{H}$ such that

$$
\left\langle\rho\left(h_{1}\right), \rho\left(h_{2}\right)\right\rangle_{\widetilde{H}}=\left\langle h_{1}, h_{2}\right\rangle_{H} .
$$

Thus $\rho$ induces a mapping from $H^{\otimes n}$ to $\widetilde{H}^{\otimes n}$ in the following way: Let $e_{1}, \ldots, e_{k}, \ldots$ be orthonormal system of $H$. Then $\tilde{e}_{k}=\rho\left(e_{k}\right), k=1,2, \ldots$, is orthonormal system of $\widetilde{H}$. If $f=\sum a_{i_{1} \cdots i_{n}} e_{i_{1}} \otimes \cdots \otimes e_{i_{n}}$, then we define

$$
\rho^{\otimes n} f=\sum a_{i_{1} \cdots i_{n}} \tilde{e}_{i_{1}} \otimes \cdots \otimes \tilde{e}_{i_{n}} .
$$

It is easy to see that for any $f, g \in H^{\otimes n}$,

$$
\left\langle\rho^{\otimes n} f, \rho^{\otimes n} g\right\rangle_{\widetilde{H}^{\otimes n}}=\langle f, g\rangle_{H^{\otimes n}} .
$$

For any element $f=\left(f_{0}, f_{1}, \ldots, f_{n}, \ldots\right)$ in $\Phi(H)$ we define

$$
\Phi(\rho) f=\left(f_{0}, \rho\left(f_{1}\right), \ldots, \rho^{\otimes n}\left(f_{n}\right), \ldots\right) .
$$

Then it is easy to check that $\Phi(\rho)$ is an isometry between the Fock spaces $\Phi(H)$ and $\Phi(\widetilde{H})$.

Since there is an isometry between $L^{2}(\Omega, \mathcal{F}, P)$ and $\Phi(H)$, we obtain an isometry between $L^{2}(\Omega, \mathcal{F}, P)$ and $L^{2}(\widetilde{\Omega}, \widetilde{H}, \widetilde{P})$. The explicit form of this isometry may be described as follows: For any $F \in L^{2}(\Omega, \mathcal{F}, P)$,

$$
F=\sum_{n=0}^{\infty} \frac{1}{n !} I_{n}\left(f_{n}\right) .
$$

Then $\widetilde{F}=\Phi(\rho) F$ is given by

$$
\widetilde{F}=\sum_{n=0}^{\infty} \frac{1}{n !} I_{n}\left(\rho^{\otimes n} f_{n}\right) .
$$

THEOREM 2.12. - Let $\rho$ be an isometry between $H$ and $\widetilde{H}$. Then $\Phi(\rho)$ is a probability structure preserving mapping from $(\Omega, \mathcal{F}, P)$ to $(\widetilde{\Omega}, \widetilde{H}, \widetilde{P})$.

Proof. - Recall that $\mathcal{E}$ is the set of finite linear combinations of $\varepsilon(h):=\exp (\langle\cdot, h\rangle-$ $\left.\|h\|_{H}^{2}\right), h \in H$ and that $\mathcal{E}$ is an algebra and a dense subset of $L^{2}(\Omega, \mathcal{F}, P)$. It is easy to verify that $\mathbb{E}(F)=\widetilde{\mathbb{E}}(\Phi(\rho) F)$ and $\Phi(\rho)(F+G)=\Phi(\rho) F+\Phi(\rho) G$. We need to prove 
the product preserving property (2.4). It is easy to see that

$$
\varepsilon(h)=\sum_{n=0}^{\infty} \frac{1}{n !} I_{n}\left(h^{\otimes n}\right) .
$$

Thus

$$
\Phi(\rho) \varepsilon(h)=\sum_{n=0}^{\infty} \frac{1}{n !} I_{n}\left(\rho(h)^{\otimes n}\right)=\varepsilon(\rho(h)) .
$$

On the other hand, it is easy to verify that

$$
\begin{aligned}
\varepsilon\left(h_{1}\right) \varepsilon\left(h_{2}\right) & =\exp \left[\left\langle\cdot, h_{1}+h_{2}\right\rangle-\left\|h_{1}\right\|_{H}^{2}-\left\|h_{2}\right\|_{H}^{2}\right] \\
& =\varepsilon\left(h_{1}+h_{2}\right) \exp \left(\left\langle h_{1}, h_{2}\right\rangle_{H}\right) .
\end{aligned}
$$

Thus

$$
\begin{aligned}
\Phi(\rho)\left[\varepsilon\left(h_{1}\right) \varepsilon\left(h_{2}\right)\right] & =\exp \left[\left\langle h_{1}, h_{2}\right\rangle_{H}\right] \Phi(\rho)\left[\varepsilon\left(h_{1}+h_{2}\right)\right] \\
& =\exp \left[\left\langle h_{1}, h_{2}\right\rangle_{H}\right] \varepsilon\left(\rho\left(h_{1}+h_{2}\right)\right) \\
& =\exp \left[\left\langle\rho\left(h_{1}\right), \rho\left(h_{2}\right)\right\rangle_{H}\right] \varepsilon\left(\rho\left(h_{1}\right)+\rho\left(h_{2}\right)\right) \\
& =\varepsilon\left(\rho\left(h_{1}\right)\right) \varepsilon\left(\rho\left(h_{2}\right)\right) \\
& =\left[\Phi(\rho) \varepsilon\left(h_{1}\right)\right]\left[\Phi(\rho) \varepsilon\left(h_{2}\right)\right] .
\end{aligned}
$$

From this the theorem follows.

Remark 2.13. - We shall call $\Phi(\rho)$ the probability structure preserving mapping induced by $\rho$.

LEMMA 2.14. - If $\rho$ is an isometry from the Hilbert space $H$ to the Hilbert space $\widetilde{H}$ and if $V=\Phi(\rho)$ is the probability structure preserving mapping induced by $\rho$, then for any $F, G \in \mathcal{E}$,

$$
V(F \diamond G)=(V F) \diamond(V G),
$$

where diamond denotes the Wick product (see [5]).

Proof. - Let $F=\varepsilon\left(h_{1}\right)$ and $G=\varepsilon\left(h_{2}\right)$, where $h_{1}, h_{2} \in H$. We have

$$
F \diamond G=\varepsilon\left(h_{1}+h_{2}\right) .
$$

Hence

$$
V(F \diamond G)=\varepsilon\left(\rho\left(h_{1}+h_{2}\right)\right)=\varepsilon\left(\rho\left(h_{1}\right)\right) \diamond \varepsilon\left(\rho\left(h_{2}\right)\right)=(V F) \diamond(V G) .
$$

This proves the lemma through a linearity argument.

\section{Stochastic integral for fractional Brownian motions}

Let us recall some results from [19] and [11]. From now on $(\Omega, \mathcal{F}, P)$ will be the classical canonical Wiener space and $(\widetilde{\Omega}, \widetilde{\mathcal{F}}, \widetilde{P})=\left(\Omega, \mathcal{F}, P^{H}\right)$ will be the canonical space for fractional Brownian motion with Hurst parameter $H \in(0,1)$. 
Fractional integrals of order $\alpha \in(0,1)$ (of Riemann-Liouville type) of a function $f$ on $\mathbb{R}$ are defined as

$$
I_{ \pm}^{\alpha} f(x)=\frac{1}{\Gamma(\alpha)} \int_{0}^{\infty} t^{\alpha-1} f(x \pm t) \mathrm{d} t
$$

where $\Gamma(x)$ is the gamma function. They are also called fractional integral of Weyl type in [14]. When $\alpha=-\beta$ is negative, $I_{ \pm}^{\alpha}$ will be the fractional derivatives (of Marchaud type)

$$
I_{ \pm}^{\alpha} f(x)=\mathrm{D}_{ \pm}^{\beta} f(x)=\frac{\beta}{\Gamma(1-\beta)} \int_{0}^{\infty} \frac{f(x)-f(x \mp t)}{t^{1+\beta}} \mathrm{d} t .
$$

We denote by

$$
\hat{f}(\xi)=\mathcal{F}(f)(\xi)=\int_{\mathbb{R}} \mathrm{e}^{\mathrm{i} x \xi} f(x) \mathrm{d} x, \quad \xi \in \mathbb{R}
$$

the Fourier transform of a function $f$. The following lemma is from [19], Theorem 7.1.

LEMmA 3.1. - If $0<\alpha<1$ and $f \in L^{1}(\mathbb{R})$, then the Fourier transform of $I_{ \pm}^{\alpha} f$ is given by

$$
\mathcal{F}\left(I_{ \pm}^{\alpha} f\right)(\xi)=(\mp \mathrm{i} \xi)^{-\alpha} \hat{f}(\xi), \quad \xi \in \mathbb{R}
$$

where

$$
(\mp \mathrm{i} \xi)^{-\alpha}=|\xi|^{-\alpha} \mathrm{e}^{\mp \frac{\alpha \pi \mathrm{i}}{2} \operatorname{sign} \xi} .
$$

Denote by $\mathcal{S}(\mathbb{R})$ the Schwartz space of rapidly decreasing functions.

From the definition of $I_{ \pm}^{\alpha} f$ and from this lemma it follows easily that if $f \in \mathcal{S}(\mathbb{R})$, then (3.3) holds for any real number $\alpha$.

The following equation holds ([19], Eqs. (5.16) and (5.17)):

$$
\int_{\mathbb{R}} g(x) I_{ \pm}^{\alpha} f(x) \mathrm{d} x=\int_{\mathbb{R}} f(x) I_{\mp}^{\alpha} g(x) \mathrm{d} x
$$

for all $f, g \in \mathcal{S}(\mathbb{R})$.

If $f \in \mathcal{S}(\mathbb{R})$ and $I_{-}^{\alpha}(f)=0$, then by (3.3) we see that

$$
(\mathrm{i} \xi)^{\alpha} \hat{f}(\xi)=0 .
$$

Thus $\hat{f}(\xi)=0$ for almost all $\xi \in \mathbb{R}$. We have then $f=0$. Denote

$$
\mathcal{S}_{-}^{\alpha}(\mathbb{R})=I_{-}^{\alpha}(\mathcal{S}(\mathbb{R}))=\left\{I_{-}^{\alpha}(f), f \in \mathcal{S}(\mathbb{R})\right\} .
$$

Then $I_{-}^{\alpha}$ is a bijective linear transformation from $\mathcal{S}(\mathbb{R})$ to $\mathcal{S}_{-}^{\alpha}(\mathbb{R})$.

For any two elements $f$ and $g$ of $\mathcal{S}_{-}^{\alpha}(\mathbb{R})$, define

$$
\langle f, g\rangle_{\Theta_{H}}:=\frac{1}{2 \pi} \int_{\mathbb{R}}|\xi|^{1-2 H} \hat{f}(\xi) \hat{g}(\xi) \mathrm{d} \xi, \quad \forall f, g \in \mathcal{S}(\mathbb{R}) .
$$


It is easy to verify that $\langle\cdot, \cdot\rangle_{\Theta_{H}}$ is an inner product on $\mathcal{S}_{-}^{\alpha}(\mathbb{R})$. Then $\mathcal{S}_{-}^{\alpha}(\mathbb{R})$ is a pre-Hilbert space with respect to the Hilbert norm induced by this inner product:

$$
\|f\|_{\Theta_{H}}=\sqrt{\langle f, g\rangle_{\Theta_{H}}}=\sqrt{\int_{\mathbb{R}}|\xi|^{1-2 H}|\hat{f}(\xi)|^{2} \mathrm{~d} \xi} .
$$

Let $\Theta_{H}$ denote its completion with respect to the Hilbert norm $\|\cdot\|_{\Theta_{H}}$. Therefore, $\Theta_{H}$ is a Hilbert space. For any $f \in \mathcal{S}(\mathbb{R})$ denote $g(x)=\left(I_{-}^{\frac{1}{2}-H} f\right)(x), x \in \mathbb{R}$. Then by (3.3)

$$
\hat{g}(\xi)=(\mathrm{i} \xi)^{H-\frac{1}{2}} \hat{f}(\xi) .
$$

From the definition of the norm $\|\cdot\|_{\Theta_{H}}$ and Parserval identity

$$
\begin{aligned}
\|g\|_{\Theta_{H}}^{2} & =\frac{1}{2 \pi} \int_{\mathbb{R}}|\xi|^{1-2 H}|\hat{g}(\xi)|^{2} \mathrm{~d} \xi=\frac{1}{2 \pi} \int_{\mathbb{R}}|\xi|^{1-2 H}\left|(\mathrm{i} \xi)^{H-\frac{1}{2}} \hat{f}(\xi)\right|^{2} \mathrm{~d} \xi \\
& =\frac{1}{2 \pi} \int_{\mathbb{R}}|\hat{f}(\xi)|^{2} \mathrm{~d} \xi=\int_{\mathbb{R}}|f(x)|^{2} \mathrm{~d} x=\|f\|_{L^{2}(\mathbb{R})}^{2} .
\end{aligned}
$$

This means that

$$
\left\|I_{-}^{\frac{1}{2}-H} f\right\|_{\Theta_{H}}=\|f\|_{L^{2}(\mathbb{R})}, \quad \forall f \in \mathcal{S}(\mathbb{R}) .
$$

Since $\mathcal{S}(\mathbb{R})$ is dense in $L^{2}(\mathbb{R})$ and $\mathcal{S}_{-}^{\alpha}(\mathbb{R})$ is dense in $\Theta_{H}$ (by the definition of $\Theta_{H}$ ) we can extend $I_{-}^{\frac{1}{2}-H}$ to an isometry between $L^{2}(\mathbb{R})$ and $\Theta_{H}$.

Namely, we have

LEMMA 3.2. $-I_{-}^{1 / 2-H}$ can be extended to an isometry from $L^{2}(\mathbb{R})$ to $\Theta_{H}$.

It is interesting to say more about the Hilbert space $\Theta_{H}$.

PROPOSITION 3.3. $-\mathcal{S}(\mathbb{R})$ is a dense subset of $\Theta_{H}$.

Proof. - Let $g$ be an element of $\mathcal{S}(\mathbb{R})$ and define $f=I_{-}^{H-\frac{1}{2}} g$. Then

$$
\hat{f}(\xi)=(\mathrm{i} \xi)^{\frac{1}{2}-H} \hat{g}(\xi) \text {. }
$$

It is clear that

$$
\int_{\mathbb{R}}|\hat{f}(\xi)|^{2} \mathrm{~d} \xi=\int_{\mathbb{R}}|\xi|^{1-2 H}|\hat{g}(\xi)|^{2} \mathrm{~d} \xi<\infty
$$

since $g \in \mathcal{S}(\mathbb{R})$ and $H<1$. Thus $f \in L^{2}(\mathbb{R})$. But

$$
\mathcal{F}\left(I^{1 / 2-H} f\right)(\xi)=(\mathrm{i} \xi)^{H-1 / 2}(\mathrm{i} \xi)^{1 / 2-H} \hat{g}(\xi)=\hat{g}(\xi),
$$

where $\mathcal{F}(g)$ denotes the Fourier transform of $g$. This means that $I^{1 / 2-H} f=g$. This implies that $\mathcal{S}(\mathbb{R})$ is a subset of $\Theta_{H}$. 
Now we need to show that $\mathcal{S}(\mathbb{R})$ is dense in $\Theta_{H}$. First let us assume that $H<1 / 2$. Let $\phi \in C^{\infty}(\mathbb{R})$ be a positive smooth function with compact support and such that

$$
\int_{\mathbb{R}} \phi(x) \mathrm{d} x=1 .
$$

Denote

$$
h(t)= \begin{cases}0 & t>0, \\ |t|^{-H-\frac{1}{2}} / \Gamma\left(\frac{1}{2}-H\right) & t<0,\end{cases}
$$

and

$$
\phi_{\varepsilon}(x)=\frac{\phi(x / \varepsilon)}{\varepsilon}, \quad \varepsilon>0, x \in \mathbb{R} .
$$

Therefore $\phi_{\varepsilon}$ is $C^{\infty}$ and with compact support (hence $\phi_{\varepsilon} \in \mathcal{S}(\mathbb{R})$ ). Consider

$$
h_{\varepsilon}(t)=\phi_{\varepsilon} * h:=\int_{\mathbb{R}} \phi_{\varepsilon}(x) h(t-x) \mathrm{d} x,
$$

where and in what follows $*$ denote the convolution. Then it is easy to see that $h_{\varepsilon}$ is an element of $\mathcal{S}(\mathbb{R})$. For any function $f \in \mathcal{S}(\mathbb{R})$, define

$$
f_{\varepsilon}(x)=\left(h_{\varepsilon} * f\right)(x) .
$$

Then $f_{\varepsilon}$ is an element of $\mathcal{S}(\mathbb{R})$. On the other hand we have

$$
f_{\varepsilon}=h_{\varepsilon} * f=\left(\phi_{\varepsilon} * h\right) * f=\phi_{\varepsilon} *(h * f) .
$$

By Eq. (5.4) of [19], we see that

$$
h * f=I^{1 / 2-H} f .
$$

Namely,

$$
f_{\varepsilon}=\phi_{\varepsilon} *\left(I^{1 / 2-H} f\right) .
$$

Applying the Fourier transformation, we obtain

$$
\hat{f}_{\varepsilon}(\xi)=\hat{\phi}_{\varepsilon}(\xi)(\mathrm{i} \xi)^{H-1 / 2} \hat{f}(\xi) .
$$

It is easy to verify that

$$
\hat{\phi}_{\varepsilon}(\xi) \rightarrow 1 \text { and }\left|\hat{\phi}_{\varepsilon}(\xi)\right| \leqslant 1
$$

for almost all $\xi$. Thus by Lebesgue's dominate convergence theorem, we see that

$$
\hat{f}_{\varepsilon}(\xi)=\hat{\phi}_{\varepsilon}(\xi)(\mathrm{i} \xi)^{H-1 / 2} \hat{f}(\xi)
$$


converges to $(\mathrm{i} \xi)^{H-1 / 2} \hat{f}(\xi)$ in $L^{2}(\mathbb{R})$ as $\varepsilon \rightarrow 0$. Hence $f_{\varepsilon}$ converges to $I^{1 / 2-H} f$ in $\Theta_{H}$ as $\varepsilon \rightarrow 0$. This implies that $\mathcal{S}(\mathbb{R})$ is dense in $\Theta_{H}$. When $H>1 / 2$, we need to use $I^{1 / 2-H}=I^{3 / 2-H} \frac{\mathrm{d}}{\mathrm{d} x}$. In this case we define

$$
h(t)= \begin{cases}0 & t>0, \\ |t|^{\frac{1}{2}-H} / \Gamma\left(\frac{3}{2}-H\right) & t<0,\end{cases}
$$

and

$$
h_{\varepsilon}(t)=\phi_{\varepsilon} * h=\int_{\mathbb{R}} \phi_{\varepsilon}(x) h(t-x) \mathrm{d} x,
$$

which is an element of $\mathcal{S}(\mathbb{R})$, where $\phi_{\varepsilon}$ is defined as above. For any function $f \in \mathcal{S}(\mathbb{R})$, define

$$
f_{\varepsilon}(x)=\left(h_{\varepsilon} * f^{\prime}\right)(x),
$$

where $f^{\prime}$ is the derivative of $f$. Then $f_{\varepsilon}$ is an element of $\mathcal{S}(\mathbb{R})$. On the other hand we have

$$
f_{\varepsilon}=h_{\varepsilon} * f=\left(\phi_{\varepsilon} * h\right) * f^{\prime}=\phi_{\varepsilon} *\left(h * f^{\prime}\right) .
$$

By Eq. (5.4) of [19], we see that

$$
h * f=I^{3 / 2-H} f^{\prime}=I^{\frac{1}{2}-H} f .
$$

Namely,

$$
f_{\varepsilon}=\phi_{\varepsilon} *\left(I^{1 / 2-H} f\right) .
$$

A similar argument can be applied to show that $f_{\varepsilon}$ converges to $I^{1 / 2-H} f$ in $\Theta_{H}$ as $\varepsilon \rightarrow 0$. This implies that $\mathcal{S}(\mathbb{R})$ is dense in $\Theta_{H}$ in the case $H>1 / 2$.

From the above argument, we also conclude that the isometry $I_{-}^{1 / 2-H}: L^{2}(\mathbb{R}) \rightarrow \Theta_{H}$ has the inverse which coincides with $I_{-}^{H-1 / 2}$ on $\mathcal{S}(\mathbb{R})$. We denote this inverse by $I_{-}^{H-1 / 2}$, which is an isometry from $\Theta_{H}$ to $L^{2}(\mathbb{R})$.

In [18], some other types of space were introduced. For example, it is denoted that

$$
\begin{aligned}
\Lambda_{H} & =\left\{f: \int_{\mathbb{R}}\left[\left(I_{-}^{H-1 / 2} f\right)(s)\right]^{2} \mathrm{~d} s<\infty\right\} \\
& =\left\{f: \int_{\mathbb{R}}\left[\int_{\mathbb{R}} f(u)(u-s)_{+}^{H-3 / 2}\right]^{2} \mathrm{~d} s<\infty\right\},
\end{aligned}
$$

when $H>1 / 2$ and

$$
\Lambda_{H}=\left\{f: \exists \phi \in L^{2}(\mathbb{R}) \text { such that } f=I_{-}^{1 / 2-H} \phi\right\}
$$

when $H<1 / 2$. The property of these and other relevant spaces are studied also. It is clear from Theorem 3.2 and Theorem 3.3 of [18] that $\Lambda_{H}$ is a subset of $\Theta_{H}$. However, $\Lambda_{H}$ is not appropriate in our paper since it is not a Hilbert space. 
In recent years, there has been a boom of study of a particular family of Gaussian processes, called fractional Brownian motions. Fractional Brownian motion with Hurst parameter $H \in(0,1)$ has been introduced by Mandelbrot and Van Ness as the fractional derivative of Brownian motion. More precisely, $\mathrm{fBm} B^{H}=\left(B_{t}^{H}, t \in \mathbb{R}_{+}\right)$with Hurst parameter $H \in(0,1)$ is defined as

$$
\begin{aligned}
B_{t}^{H}= & \frac{1}{\Gamma(H+1 / 2)} \int_{-\infty}^{0}\left[(t-s)^{H-1 / 2}-(-s)^{H-1 / 2}\right] \mathrm{d} B_{s} \\
& +\int_{0}^{t}(t-s)^{H-1 / 2} \mathrm{~d} B_{s}, \quad t \in \mathbb{R}_{+},
\end{aligned}
$$

where $B=\left(B_{s}, s \in \mathbb{R}\right)$ is a Wiener process on some probability space $(\Omega, \mathcal{F}, P)$. This probability space will be fixed. The expectation on $(\Omega, \mathcal{F}, P)$ is denoted by $\mathbb{E}$. The fractional Brownian motion satisfies

$$
\mathbb{E}\left(B_{t}^{H} B_{s}^{H}\right)=c_{H}\left(|t|^{2 H}+|s|^{2 H}-|t-s|^{2 H}\right),
$$

where $c_{H}=\frac{\Gamma(2-2 H) \cos (\pi H)}{\pi H(1-H)}$.

As in [11], heuristically we may write (3.5) as

$$
B_{t}^{H}=I_{+}^{H+1 / 2}(\dot{B})(t)-I_{+}^{H+1 / 2}(\dot{B})(0) .
$$

[Each of these two terms may not be well-defined.] Thus we have

$$
\dot{B}_{t}^{H}=\frac{\mathrm{d}}{\mathrm{d} t} I_{+}^{H+1 / 2}(\dot{B})(t)=I_{+}^{H-1 / 2}(\dot{B})(t) .
$$

Hence formally, $\dot{B}^{H}$ is the fractional integral of order $H-1 / 2$ of the white noise $\dot{B}$ when $H>1 / 2$ and $\dot{B}^{H}$ is the fractional derivative of order $1 / 2-H$ of the white noise $\dot{B}$ when $H<1 / 2$.

Using the adjoint operator (i.e., $I_{-}^{H-1 / 2}$ ) of $I_{+}^{H-1 / 2}$, we know that if $f \in \Theta_{H}$, then $\int_{\mathbb{R}} f(t) \mathrm{d} B_{t}^{H}$ may be well defined by

$$
\int_{\mathbb{R}} f(t) \mathrm{d} B_{t}^{H}=\int_{\mathbb{R}} I_{-}^{H-1 / 2}(f)(t) \mathrm{d} B_{t} .
$$

It is easy to see that

$$
\mathbb{E}\left(\int_{\mathbb{R}} f(t) \mathrm{d} B_{t}^{H}\right)=0, \quad \mathbb{E}\left(\int_{\mathbb{R}} f(t) \mathrm{d} B_{t}^{H} \int_{\mathbb{R}} g(t) \mathrm{d} B_{t}^{H}\right)=\langle f, g\rangle_{\Theta_{H}} .
$$

(See [11] for more discussion.)

From now on we denote by $V$ the probability structure preserving mapping between $L^{2}(\Omega, \mathcal{F}, P)$ and $L^{2}\left(\Omega, \mathcal{F}, P^{H}\right)$ induced by $I_{-}^{1 / 2-H}$.

Eq. (3.6) can also be used to define the stochastic integral for general random kernel. 
DEFINITION 3.4. - Let $f: \mathbb{R} \times \Omega \rightarrow \mathbb{R}$ be a stochastic process not necessarily adapted. Assume that for almost every $\omega \in \Omega, f(\cdot, \omega) \in \Theta_{H}$. If $\int_{\mathbb{R}} I_{-}^{1 / 2-H} V^{-1} f(t) \mathrm{d} B_{t}$ exists as a random variable, then we say that $\int_{\mathbb{R}} f(t) \mathrm{d} B_{t}^{H}$ exists and define the anticipative stochastic integral by

$$
\int_{\mathbb{R}} f(t) \mathrm{d} B_{t}^{H}=V\left(\int_{\mathbb{R}} I_{-}^{H-1 / 2}\left(V^{-1} f\right)(t) \mathrm{d} B_{t}\right) .
$$

PROPOSITION 3.5. - If $H>1 / 2$ and if $\int_{\mathbb{R}} f(t) \mathrm{d} B_{t}^{H}$ is well-defined in the sense of [5], then this definition (3.7) coincides with the definition introduced in [5].

Proof. - Let $F$ be an exponential function on $\left(\Omega, \mathcal{F}, P^{H}\right)$. Consider $f(t)=\chi_{(a, b]}(t) F$, $t \in \mathbb{R}$, where $-\infty<a<b<\infty$ are given numbers. Then by the definition of [5], we have $\int_{\mathbb{R}} f(t) \mathrm{d} \widetilde{B}_{t}^{H}=F \diamond\left(B_{b}^{H}-B_{a}^{H}\right)$, where $\diamond$ denotes the Wick product. Here we temporarily denote the stochastic integral in [5] by $\int_{\mathbb{R}} f(t) \tilde{\mathrm{d}} B_{t}^{H}$. On the other hand, by (3.7),

$$
\begin{aligned}
\int_{\mathbb{R}} f(t) \mathrm{d} B_{t}^{H} & =V\left(\int_{\mathbb{R}}\left(V^{-1} F\right)\left[I_{-}^{H-1 / 2} \chi_{(a, b]}\right](t) \mathrm{d} B_{t}\right) \\
& =V\left(V^{-1} F \diamond \int_{\mathbb{R}}\left[I_{-}^{H-1 / 2} \chi_{(a, b]}\right](t) \mathrm{d} B_{t}\right) \\
& =F \diamond \int_{\mathbb{R}} \chi_{(a, b]}(t) \mathrm{d} B_{t}^{H}=F \diamond\left(B_{b}^{H}-B_{a}^{H}\right) .
\end{aligned}
$$

Hence $\int_{\mathbb{R}} f(t) \tilde{\mathrm{d}} B_{t}^{H}=\int_{\mathbb{R}} f(t) \mathrm{d} B_{t}^{H}$ for step functions. The proposition follows from linearity and a limiting argument.

Let $e_{1}, \ldots, e_{k}, \ldots$ be an ONB of $L^{2}(\mathbb{R})$ such that $e_{k} \in \mathcal{S}(\mathbb{R}), k=1,2, \ldots$ Then $\left\{\tilde{e}_{1}, \tilde{e}_{2}, \ldots\right\}$ is an ONB of $\Theta_{H}$. The Malliavin derivative $D_{s}$ of a smooth functional $F=f\left(\int_{\mathbb{R}} e_{1}(t) \mathrm{d} B_{t}, \ldots, \int_{\mathbb{R}} e_{k}(t) \mathrm{d} B_{t}\right)$ is defined as

$$
D_{s} F=\sum_{n=1}^{k} \frac{\partial f}{\partial x_{n}}\left(\int_{\mathbb{R}} e_{1}(t) \mathrm{d} B_{t}, \ldots, \int_{\mathbb{R}} e_{k}(t) \mathrm{d} B_{t}\right) e_{n}(s) .
$$

Now let $G=g\left(\int_{\mathbb{R}} \tilde{e}_{1}(t) \mathrm{d} B_{t}^{H}, \ldots, \int_{\mathbb{R}} \tilde{e}_{k}(t) \mathrm{d} B_{t}^{H}\right)$. We define the derivative $D_{s}^{H}$ by

$$
D_{s}^{H} G=\sum_{n=1}^{k} \frac{\partial f}{\partial x_{n}}\left(\int_{\mathbb{R}} \tilde{e}_{1}(t) \mathrm{d} B_{t}^{H}, \ldots, \int_{\mathbb{R}} \tilde{e}_{k}(t) \mathrm{d} B_{t}^{H}\right) \tilde{e}_{n}(s) .
$$

In the case of no ambiguity (as it usually is) we omit the dependence on $H$ in $D_{t}^{H}$.

As in [15] (see also [13,17]) we denote by $\mathbb{L}^{1,2}(\Omega, \mathcal{F}, P$ ) the class of processes $u \in L^{2}(T \times \Omega)$ on the probability space $(\Omega, \mathcal{F}, P)$ such that $u(t) \in D^{1,2}$ for almost all $t$, and that

$$
\int_{\mathbb{R}^{2}}\left(D_{s} u(t)\right)^{2} \mathrm{~d} s \mathrm{~d} t<\infty
$$


This means that

$$
\mathbb{L}^{1,2}(\Omega, \mathcal{F}, P)=\left\{f: \int_{\mathbb{R}} \mathbb{E}|f(t)|^{2} \mathrm{~d} t+\int_{\mathbb{R}^{2}} \mathbb{E}\left|D_{s} f(t)\right|^{2} \mathrm{~d} s \mathrm{~d} t<\infty\right\} .
$$

The following proposition will be useful.

Proposition 3.6. - Let $f \in \mathbb{L}^{1,2}(\Omega, \mathcal{F}, P)$. Then

$$
V\left(\int_{\mathbb{R}} f(t) \mathrm{d} B_{t}\right)=\int_{\mathbb{R}}\left[I_{-}^{1 / 2-H}(V f)\right](t) \mathrm{d} B_{t}^{H} .
$$

Proof. - Since for a.a. $\omega \in \Omega, f(\cdot, \omega) \in L^{2}(\mathbb{R}) . V f(\cdot, \omega) \in L^{2}(\mathbb{R})$. Thus

$$
g(\cdot, \omega):=I_{-}^{\frac{1}{2}-H}(V f)(\cdot, \omega) \in \Theta_{H}
$$

and

$$
I_{-}^{H-\frac{1}{2}} g(\cdot, \omega)=(V f)(\cdot, \omega) .
$$

Consequently,

$$
I_{-}^{H-\frac{1}{2}}\left(V^{-1} g\right)=f
$$

By definition (3.4) we obtain

$$
\begin{aligned}
\int_{\mathbb{R}}\left(I_{-}^{1 / 2-H}\right)(V f)(t) \mathrm{d} B_{t}^{H} & =\int_{\mathbb{R}} g(t) \mathrm{d} B_{t}^{H}=V\left(\int_{\mathbb{R}} I_{-}^{H-1 / 2}\left(V^{-1} g\right)(t) \mathrm{d} B_{t}\right) \\
& =V\left(\int_{\mathbb{R}} f(t) \mathrm{d} B_{t}\right) .
\end{aligned}
$$

This proves the proposition.

Lemma 3.7. - Let $F \in \mathbb{L}^{1,2}(\Omega, \mathcal{F}, P)$. Then

$$
V D_{s} F=I_{-}^{H-1 / 2}(D)_{s}(V F),
$$

where $I_{-}^{H-1 / 2}\left(D^{H}\right)_{S} G$ denotes the application of $I_{-}^{H-1 / 2}$ to $D_{s}^{H} G$ (as a function of $s$ ).

Proof. - Let $F=\exp \left(\int_{\mathbb{R}} h(s) \mathrm{d} B_{s}\right)$. Then

$$
D_{s} F=h(s) F .
$$

Thus

$$
V D_{s} F=h(s) \exp \left(\int_{\mathbb{R}} I_{-}^{1 / 2-H} h(s) \mathrm{d} B_{s}^{H}\right)
$$


On the other hand, we have

$$
V F=\exp \left(\int_{\mathbb{R}} I_{-}^{1 / 2-H} h(s) \mathrm{d} B_{s}^{H}\right) .
$$

Hence

$$
D_{s}^{H} V F=I_{-}^{1 / 2-H} h(s) \exp \left(\int_{\mathbb{R}} I_{-}^{1 / 2-H} h(s) \mathrm{d} B_{s}^{H}\right) .
$$

This proves that $V D_{s} F=I_{-}^{H-1 / 2}\left(D^{H}\right)_{s} V F$ for exponential functions. By linearity of $V$ and $D_{s}$, we can conclude that (3.9) is true for all $F \in \mathcal{E}$. The theorem is proved by a limiting argument.

LEMMA 3.8. - Let $\Gamma: \Omega \rightarrow \Omega$ and $\Lambda: \widetilde{\Omega} \rightarrow \widetilde{\Omega}$ be measurable mappings such that the diagram (2.10) commutes. Let $V(F \circ \Gamma)=(V F) \circ \Lambda$ for any $F \in \mathcal{E}$. Then for all $F \in \mathcal{E}$,

$$
V\left(F \circ \Gamma^{-1}\right)=(V F) \circ \Lambda^{-1} .
$$

Proof. - From $F=F \circ \Gamma^{-1} \circ \Gamma$, it follows that

$$
V \circ F=V \circ\left(F \circ \Gamma^{-1} \circ \Gamma\right)=V \circ\left(F \circ \Gamma^{-1}\right) \circ \Lambda .
$$

Thus $V \circ\left(F \circ \Gamma^{-1}\right)=(V \circ F) \circ \Lambda^{-1}$.

LEMMA 3.9. - Let the assumptions of Lemma 3.8 be satisfied and let $\Gamma$ be differentiable in the sense that for any $F \in \mathbb{L}^{1,2}, F \circ \Gamma$ is in $\mathbb{L}^{1,2}$. Then

$$
V\left(D_{s}\left(F \circ \Gamma^{-1}\right) \circ \Gamma\right)=I_{-}^{H-1 / 2}\left(D^{H}\right)_{s}\left[(V F) \circ \Lambda^{-1}\right] \circ \Lambda .
$$

Proof. - From Lemmas 3.7 and 3.8, it follows that

$$
\begin{aligned}
V\left(D_{s}\left(F \circ \Gamma^{-1}\right) \circ \Gamma\right) & =\left[V D_{s}\left(F \circ \Gamma^{-1}\right)\right] \circ \Lambda \\
& =I_{-}^{H-1 / 2}\left(D^{H}\right)_{s}\left(V\left(F \circ \Gamma^{-1}\right)\right) \circ \Lambda \\
& =I_{-}^{H-1 / 2}\left(D^{H}\right)_{s}\left[(V F) \circ \Lambda^{-1}\right] \circ \Lambda .
\end{aligned}
$$

This proves the lemma.

\section{Application to absolute continuity}

Now consider an (anticipative) translation of $B^{H}$

$$
\Lambda: \quad B^{H}+\int_{0} f\left(s, B^{H}\right) \mathrm{d} s
$$


Define an anticipative translation of $B$ by

$$
\Gamma: \quad B .+\int_{0} g(s, B) \mathrm{d} s,
$$

where $g=I_{+}^{1 / 2-H} V^{-1} f$. Thus $f$ can also be computed from $g$ by $f=I_{+}^{H-1 / 2} V g$.

LEMMA 4.1. - The following diagram

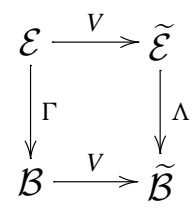

commutes, where $\mathcal{E}$ is the set of finite linear combinations of exponential functionals on $(\Omega, \mathcal{F}, P)$ and $\widetilde{\mathcal{E}}$ is the set of finite linear combinations of exponential functionals on $\left(\Omega, \mathcal{F}, P^{H}\right)$ and $\mathcal{B}=\Gamma \mathcal{E}$ and $\widetilde{\mathcal{B}}=\Lambda \widetilde{\mathcal{E}}$.

Proof. - Denote $\tilde{g}=V^{-1} f$. Let $F=\exp \left(\int_{\mathbb{R}} h(s) \mathrm{d} B_{s}\right)$, where $h \in L^{2}(\mathbb{R})$. As discussed before we denote $T=I_{-}^{1 / 2-H}$. Then

$$
\begin{aligned}
\Gamma F & =\exp \left[\int_{\mathbb{R}} h(s) \mathrm{d} B_{s}+\int_{\mathbb{R}} h(s) g(s) \mathrm{d} s\right] \\
& =\exp \left[\int_{\mathbb{R}} h(s) \mathrm{d} B_{s}+\int_{\mathbb{R}} h(s) I_{+}^{1 / 2-H} \tilde{g}(s) \mathrm{d} s\right] \\
& =\exp \left[\int_{\mathbb{R}} h(s) \mathrm{d} B_{s}+\int_{\mathbb{R}}(T h)(s) \tilde{g}(s) \mathrm{d} s\right] .
\end{aligned}
$$

Thus

$$
\begin{aligned}
V \Gamma F & =\exp \left[\int_{\mathbb{R}}(T h)(s) \mathrm{d} B_{s}^{H}+\int_{\mathbb{R}}(T h)(s) V \tilde{g}(s) \mathrm{d} s\right] \\
& =\exp \left[\int_{\mathbb{R}}(T h)(s) \mathrm{d} B_{s}^{H}+\int_{\mathbb{R}}(T h)(s) f(s) \mathrm{d} s\right] .
\end{aligned}
$$

On the other hand,

$$
V F=\exp \left[\int_{\mathbb{R}}(T f)(s) \mathrm{d} B_{s}^{H}\right]
$$

Therefore, we have

$$
\Lambda V F=\exp \left[\int_{\mathbb{R}}(T h)(s) \mathrm{d} B_{s}^{H}+\int_{\mathbb{R}}(T h)(s) f(s) \mathrm{d} s\right] .
$$


This shows that the diagram commute for exponential functionals. The lemma is completed by a linearity argument.

LemmA 4.2. - Let $\Gamma: \Omega \rightarrow \Omega$ and $\Lambda: \widetilde{\Omega} \rightarrow \widetilde{\Omega}$ be defined by (4.1) and (4.2). Then for all measurable $F$,

$$
V(F \circ \Gamma)=(V F) \circ \Lambda .
$$

Proof. - Let $F=\exp \left\{\int h(s) \mathrm{d} B_{s}\right\}$. Then

$$
\begin{aligned}
F \circ \Gamma & =\exp \left\{\int h(s) \mathrm{d} B_{s}+\int h(s) I_{+}^{1 / 2-H} V^{-1} f(s) \mathrm{d} s\right\} \\
& =\exp \left\{\int h(s) \mathrm{d} B_{s}+\int\left(I_{-}^{1 / 2-H} h\right)(s) V^{-1} f(s) \mathrm{d} s\right\} .
\end{aligned}
$$

Consequently,

$$
V(F \circ \Gamma)=\exp \left\{\int\left(I_{-}^{1 / 2-H} h\right)(s) \mathrm{d} B_{s}^{H}+\int\left(I_{-}^{1 / 2-H} h\right)(s) f(s) \mathrm{d} s\right\}=(V F) \circ \Lambda .
$$

This proves the lemma for exponential functional. The lemma follows from a linearity argument.

Let us recall a result on Radon-Nikodym derivative. The following results can be found in [2] when the interval is $[0,1]$.

THEOREM 4.3. - Let $f$ and $g$ be as in (4.1)-(4.2) such that $f$ and $g$ are in $\mathbb{L}^{1,2}$. Let the following conditions be satisfied

(i) There is a positive number $\gamma \in(0,1)$ such that

$$
\int_{\mathbb{R}^{2}}\left|D_{t} g(s)\right|^{2} \mathrm{~d} s \mathrm{~d} t \text { is bounded by } \gamma .
$$

(ii) There is a positive number $q>1$ with

$$
\mathbb{E}\left[\exp \left\{\frac{q}{2} \int_{\mathbb{R}} g^{2}(s) \mathrm{d} s\right\}\right]<\infty .
$$

Then $\Gamma$ is invertible and $P \circ \Gamma^{-1}$ is absolutely continuous with respect to $P$. Moreover, the following identity is true:

$$
\frac{\mathrm{d} P \circ \Gamma^{-1}}{\mathrm{~d} P}=\kappa \exp \left\{-\int_{\mathbb{R}} g(s) \mathrm{d} B_{s}-\frac{1}{2} \int_{\mathbb{R}} g^{2}(s) \mathrm{d} s\right\},
$$

with

$$
\kappa=\exp \left\{-\int_{\mathbb{R}} \int_{0}^{s} D_{s} g(r) D_{r}\left[g\left(s, \Gamma_{s}^{-1}\right)\right] \circ \Gamma_{s}\right\},
$$


where $\Gamma_{s}$ is defined by

$$
\Gamma_{s}: B . \rightarrow B .+\int_{0}^{s \wedge} g(u) \mathrm{d} u
$$

and $\Gamma_{s}^{-1}$ is the inverse of $\Gamma_{s}$.

To obtain a Radon-Nikodym derivative for fractional Brownian motions, we define

$$
h(u):=I_{+}^{H-1 / 2}\left(I_{[0, s]} I_{+}^{1 / 2-H} f\right)(u), \quad u \in \mathbb{R}
$$

and

$$
\Lambda_{s}: B_{\cdot}^{H} \rightarrow B_{\cdot}^{H}+\int_{0} h(u) \mathrm{d} u .
$$

The main theorem of this section is

THEOREM 4.4. - Let the following conditions be satisfied

(i) There is a positive number $\gamma \in(0,1)$ such that

$$
\int_{\mathbb{R}^{2}}\left|I_{-}^{H-1 / 2}\left(D^{H}\right)_{s} I_{+}^{1 / 2-H}(f)(s)\right|^{2} \mathrm{~d} s \mathrm{~d} t \text { is bounded by } \gamma \text {. }
$$

(ii) There is a positive number $q>1$ with

$$
\mathbb{E}\left[\exp \left\{\frac{q}{2}\|f\|_{\Theta_{H}}^{2}\right\}\right]<\infty
$$

Then $\Gamma$ is invertible and $P^{H} \circ \Lambda^{-1}$ is absolutely continuous with respect to $P^{H}$. Moreover, the following identity is true:

$$
\frac{\mathrm{d} P^{H} \circ \Lambda^{-1}}{\mathrm{~d} P^{H}}=\mathcal{L}=\tilde{\kappa} \exp \left\{-\int_{\mathbb{R}} I_{-}^{1 / 2-H} I_{+}^{1 / 2-H} f(s) \mathrm{d} B_{s}^{H}-\frac{1}{2}\|f\|_{\Theta_{H}}^{2}\right\},
$$

where

$$
\begin{aligned}
\tilde{\kappa}=\exp \{ & -\int_{\mathbb{R}} \int_{0}^{s} I_{-}^{H-1 / 2}\left(D^{H}\right)_{s} I_{+}^{1 / 2-H}(f)(r) I_{-}^{H-1 / 2}\left(D^{H}\right)_{r} \\
& \left.\times\left(I_{+}^{1 / 2-H} f\right)\left(s, \Lambda_{s}\right) \circ \Lambda_{s}^{-1} \mathrm{~d} s \mathrm{~d} r\right\},
\end{aligned}
$$

where $\Lambda_{s}$ is defined by

$$
\Lambda_{s}: B_{\cdot}^{H} \rightarrow B_{\cdot}^{H}+\int_{0} h(u) \mathrm{d} u
$$


and

$$
h(u):=I_{+}^{H-1 / 2}\left(I_{[0, s]} I_{+}^{1 / 2-H} f\right)(u), \quad u \in \mathbb{R},
$$

and $\Lambda_{s}^{-1}$ is the inverse of $\Lambda_{s}$.

Proof. - By Lemma 3.7, we have

$$
\begin{aligned}
V\left\{\int_{\mathbb{R}^{2}}\left|D_{t} g(s)\right|^{2} \mathrm{~d} s \mathrm{~d} t\right\} & =V \int_{\mathbb{R}^{2}}\left|D_{t} I_{+}^{1 / 2-H} V^{-1} f\right|^{2} \mathrm{~d} s \mathrm{~d} t \\
& =\int_{\mathbb{R}^{2}}\left|V D_{t} I_{+}^{1 / 2-H} V^{-1} f\right|^{2} \mathrm{~d} s \mathrm{~d} t \\
& =\int_{\mathbb{R}^{2}}\left|I_{-}^{H-1 / 2}\left(D^{H}\right)_{t} I_{+}^{1 / 2-H} f\right|^{2} \mathrm{~d} s \mathrm{~d} t .
\end{aligned}
$$

Thus (4.11) implies that (4.5) holds. By the probability structure preserving property of $V$, we obtain

$$
\begin{aligned}
\mathbb{E}\left[\exp \left\{\frac{q}{2} \int_{\mathbb{R}} g^{2}(s) \mathrm{d} s\right\}\right] & =\mathbb{E}\left[V \exp \left\{\frac{q}{2} \int_{\mathbb{R}} g^{2}(s) \mathrm{d} s\right\}\right] \\
& =\mathbb{E}\left[\exp \left\{\frac{q}{2} \int_{\mathbb{R}}\left|I_{-}^{1 / 2-H} f(s)\right|^{2} \mathrm{~d} s\right\}\right] \\
& =\mathbb{E}\left[\exp \left\{\frac{q}{2}\|f\|_{\Theta_{H}}^{2}\right\}\right]
\end{aligned}
$$

Hence (4.12) implies that (4.6) is true. Therefore under the assumptions of Theorem 4.4, $\frac{\mathrm{d} P \circ \Gamma^{-1}}{\mathrm{~d} P}$ exists. It suffices to compute $V \circ \frac{\mathrm{d} P \circ \Lambda^{-1}}{\mathrm{~d} P}$. First let's make the following computation.

$$
\begin{aligned}
V & \exp \left\{-\int_{\mathbb{R}} g(s) \mathrm{d} B_{s}-\frac{1}{2} \int_{\mathbb{R}} g^{2}(s) \mathrm{d} s\right\} \\
& =\exp \left\{-\int_{\mathbb{R}} I_{-}^{1 / 2-H} V g(s) \mathrm{d} B_{s}^{H}-\frac{1}{2} \int_{\mathbb{R}} V g^{2}(s) \mathrm{d} s\right\} \\
& =\exp \left\{-\int_{\mathbb{R}} I_{-}^{1 / 2-H} I_{+}^{1 / 2-H} f(s) \mathrm{d} B_{s}^{H}-\frac{1}{2} \int_{\mathbb{R}}\left|I_{+}^{1 / 2-H} f\right|^{2}(s) \mathrm{d} s\right\} \\
& =\exp \left\{-\int_{\mathbb{R}} I_{-}^{1 / 2-H} I_{+}^{1 / 2-H} f(s) \mathrm{d} B_{s}^{H}-\frac{1}{2}\|f\|_{\Theta_{H}}^{2}\right\} .
\end{aligned}
$$


Next we have to evaluate $\tilde{\kappa}=V \kappa$. Similar to Lemmas 4.1 and 4.2 , we can prove that the following diagram

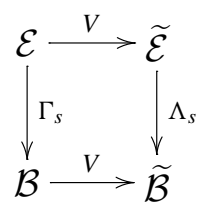

commutes and that $V\left(F \circ \Gamma_{s}\right)=(V F) \circ \Lambda_{s}$ holds. By Lemma 3.7,

$$
V\left(D_{s} g(r)\right)=I_{-}^{H-1 / 2}\left(D^{H}\right)_{s}[V g(r)]=I_{-}^{H-1 / 2}\left(D^{H}\right)_{s} I_{+}^{1 / 2-H}(f)(r) .
$$

From Lemma 3.9 it follows that

$$
\begin{aligned}
V\left(D_{r}\left(g\left(s, \Gamma_{s}^{-1}\right)\right) \circ \Gamma_{s}\right) & =I_{-}^{H-1 / 2}\left(D^{H}\right)_{r}\left[(V g) \circ \Lambda_{s}^{-1}\right] \circ \Lambda_{s} \\
& =I_{-}^{H-1 / 2}\left(D^{H}\right)_{r}\left(I_{+}^{1 / 2-H} f\right)\left(s, \Lambda_{s}^{-1}\right) \circ \Lambda_{s} .
\end{aligned}
$$

Thus

$$
\begin{aligned}
V \kappa=\exp \{ & -\int_{\mathbb{R}}^{s} \int_{0}^{s} I_{-}^{H-1 / 2}(D)_{s} I_{+}^{1 / 2-H}(f)(r) I_{-}^{H-1 / 2}(D)_{r} \\
& \left.\times\left(I_{+}^{1 / 2-H} f\right)\left(s, \Lambda_{s}\right) \circ \Lambda_{s}^{-1} \mathrm{~d} s \mathrm{~d} r\right\} .
\end{aligned}
$$

This proves the theorem.

Remark 4.5.- (a) If $f$ is deterministic, then $D_{s} f=0$. Thus $\tilde{\kappa} \equiv 1$. Therefore if $f \in \Theta_{h}$ is deterministic, then

$$
\frac{\mathrm{d} P^{H} \circ \Lambda^{-1}}{\mathrm{~d} P^{H}}=\exp \left\{-\int_{\mathbb{R}} I_{-}^{1 / 2-H} I_{+}^{1 / 2-H} f(s) \mathrm{d} B_{s}^{H}-\frac{1}{2}\|f\|_{\Theta_{H}}^{2}\right\} .
$$

By elementary results from the fractional calculus, we see that this formula coincides with the formula in [11]. This is in fact Cameron-Martin formula. See [16] for general Gaussian process case.

(b) To obtain the Girsanov formula for finite interval, one needs to compute the conditional expectation as indicated in [11].

\section{Acknowledgements}

The author is grateful for the referee's careful reading and constructive comments on the original version of the paper.

\section{REFERENCES}

[1] Alòs E., Mazet, O., Nualart D., Stochastic calculus with respect to fractional Brownian motion with Hurst parameter lesser than $\frac{1}{2}$, Stochastic Process. Appl. 86 (2000) 121-139. 
[2] Buckdahn R., Anticipative Girsanov transformations and Skorohod stochastic differential equations, Mem. Amer. Math. Soc. 111 (533) (1994).

[3] Dai W., Heyde C.C., Itô formula with respect to fractional Brownian motion and its application, J. Appl. Math. Stoch. Anal. 9 (1996) 439-448.

[4] Decreusefond L., Üstünel A.S., Stochastic analysis of the fractional Brownian motion, Potential Analysis 10 (1999) 177-214.

[5] Duncan T.E., Hu Y.Z., Pasik-Duncan B., Stochastic calculus for fractional Brownian motion, I. Theory, SIAM J. Control Optim. 38 (2000) 582-612.

[6] Hu Y.Z., Kallianpur G., Exponential integrability and application to stochastic quantization, Appl. Math. Optim. 37 (1998) 295-353.

[7] Hu Y.Z., Meyer P.A., Chaos de Wiener et intégrale de Feynman, in: Séminaire de Probabilités, XXII, Lecture Notes in Math., Vol. 1321, Springer, Berlin, 1988, pp. 51-71.

[8] Hu Y.Z., Meyer P.A., Sur les intégrales multiples de Stratonovich, in: Séminaire de Probabilités, XXII, Lecture Notes in Math., Vol. 1321, Springer, Berlin, 1988, pp. 72-81.

[9] Hu Y.Z., Meyer P.A., On the approximation of multiple Stratonovich integrals, in: Stochastic Processes, Springer, New York, 1993, pp. 141-147.

[10] Hu Y.Z., Øksendal B., Fractional white noise calculus and applications to finance, Preprint, University of Oslo, 1999.

[11] Hu Y.Z., Prediction and translation of fractional Brownian motions, in: Hida T. et al. (Eds.), Stochastics in Finite and Infinite Dimensions, Birkhäuser, 2000, pp. 153-171.

[12] Janson S., Gaussian Hilbert Spaces, Cambridge University Press, 1997.

[13] Malliavin P., Stochastic Analysis, Grundlehren der Mathematischen Wissenschaften, Vol. 313, Springer, Berlin, 1997.

[14] Mandelbrot B.B., Van Ness J.W., Fractional Brownian motions, fractional noises and applications, SIAM Rev. 10 (1968) 422-437.

[15] Nualart D., The Malliavin Calculus and Related Topics, Probability and its Applications, Springer, New York, 1995.

[16] Neveu J., Processus aléatoires gaussiens, Les Presses de l'Université de Montréal, Montreal, Quebec, 1968.

[17] Norris J., Simplified Malliavin calculus, in: Séminaire de Probabilités, XX, 1984/85, Lecture Notes in Math., Vol. 1204, Springer, Berlin, 1986, pp. 101-130.

[18] Pipiras V., Taqqu M.S., Integration questions related to fractional Brownian motion, Probab. Theory Related Fields 118 (2000) 251-291.

[19] Samko S.G., Kilbas A.A., Marichev O.I., Fractional Integrals and Derivatives, Theory and Applications, Gordon and Breach Science Publishers, 1993.

[20] Stroock D.W., Varadhan S.R.S., Multidimensional Diffusion Processes, Springer, Berlin, 1979.

[21] Üstünel A.S., Zakai M., Transformation of Measure on Wiener Space, Springer Monographs in Mathematics, Springer, Berlin, 2000. 